\title{
DIOPHANTINE EQUATIONS WITH APPELL SEQUENCES
}

\author{
ANDRÁS BAZSÓ AND ISTVÁN PINK
}

\begin{abstract}
We consider the Diophantine equation $P_{n}(x)=g(y)$ in $x, y$ where $P_{n}(x), g(x) \in \mathbb{Q}[x], \operatorname{deg} g(x) \geq 3$ and $\left\{P_{n}(x)\right\}_{n>0}$ is an Appell sequence. Under some reasonable assumptions on $P_{n}(x)$ we prove an ineffective finiteness result on the above equation.
\end{abstract}

\section{INTRODUCTION}

For $n \in \mathbb{N} \cup\{0\}$, let $P_{n}(x)$ be a polynomial with rational coefficients and with $\operatorname{deg} P_{n}(x)=n$. Further, let $P_{0}(x)$ be a non-zero constant. The sequence $\left\{P_{n}(x)\right\}_{n \geq 0}$ is called an Appell sequence (and $P_{n}(x)$ is called an Appell polynomial) if

$$
P_{n}^{\prime}(x)=n P_{n-1}(x) \quad \text { for all } n \in \mathbb{N} .
$$

The history of such polynomials goes back to Appell's work [2] in 1880. There are several well-known examples of Appell sequences, such as the Bernoulli polynomials $B_{n}(x)$, the Euler polynomials $E_{n}(x)$, and the Hermite polynomials $H_{n}(x)$, respectively defined by the following generating series (see e.g. [12])

$$
\begin{gathered}
\frac{t \exp (t x)}{\exp (t)-1}=\sum_{n=0}^{\infty} B_{n}(x) \frac{t^{n}}{n !} ; \\
\frac{2 \exp (x t)}{\exp (t)+1}=\sum_{n=0}^{\infty} E_{n}(x) \frac{t^{n}}{n !} \quad(|t|<\pi) ; \\
\frac{\exp (t x)}{\exp \left(t^{2} / 2\right)}=\sum_{n=0}^{\infty} H_{n}(x) \frac{t^{n}}{n !} .
\end{gathered}
$$

The above defined Hermite polynomials $H_{n}(x)$ are sometimes denoted by $H e_{n}(x)$, e.g. in the book of Abramowitz and Stegun [1].

The following properties of Appell polynomials will often be used in the text, sometimes without special reference.

2010 Mathematics Subject Classification. 11D41, 11B83, 05A40.

Key words and phrases. Diophantine equations, Appell sequences, decomposition. 
We recall the so-called Appell Identity:

$$
P_{n}(x+y)=\sum_{k=0}^{n}\left(\begin{array}{l}
n \\
k
\end{array}\right) P_{k}(x) y^{n-k}=\sum_{k=0}^{n}\left(\begin{array}{l}
n \\
k
\end{array}\right) P_{k}(y) x^{n-k},
$$

which, by setting $y=0$, implies that there exists a sequence of rational numbers $\left\{c_{n}\right\}_{n \geq 0}$ with $c_{0} \neq 0$ such that

$$
P_{n}(x)=\sum_{k=0}^{n}\left(\begin{array}{l}
n \\
k
\end{array}\right) c_{k} x^{n-k}, \quad \text { where } c_{k}:=P_{k}(0)(k \geq 0) .
$$

For the proofs of (2) and (3) see, for instance the book of Roman [12].

Let $\mathbb{K}$ be an arbitrary field. We denote by $\mathbb{K}[x]$ the ring of polynomials in the variable $x$ with coefficients from $\mathbb{K}$. A decomposition of a polynomial $F(x)$ over $\mathbb{K}$ is an equality of the following form

$$
F(x)=G_{1}\left(G_{2}(x)\right) \quad\left(G_{1}(x), G_{2}(x) \in \mathbb{K}[x]\right),
$$

which is nontrivial if

$$
\operatorname{deg} G_{1}(x)>1 \quad \text { and } \quad \operatorname{deg} G_{2}(x)>1 .
$$

Two decompositions $F(x)=G_{1}\left(G_{2}(x)\right)$ and $F(x)=H_{1}\left(H_{2}(x)\right)$ are said to be equivalent if there exists a linear polynomial $\ell(x) \in \mathbb{K}[x]$ such that $G_{1}(x)=H_{1}(\ell(x))$ and $H_{2}(x)=\ell\left(G_{2}(x)\right)$. The polynomial $F(x)$ is called decomposable over $\mathbb{K}$ if it has at least one nontrivial decomposition over $\mathbb{K}$; otherwise it is said to be indecomposable.

The decomposition of Bernoulli polynomials has been described by Bilu et al. in [6]. Decomposition properties of Euler polynomials were recently investigated by Rakaczki and Kreso [11]. The mentioned results can both be summarized as follows: the corresponding polynomial $\left(B_{n}(x)\right.$ or $\left.E_{n}(x)\right)$ is indecomposable over $\mathbb{C}$ for all odd $n$, while, if $n$ is even, then any nontrivial decomposition of the polynomial under consideration over $\mathbb{C}$ is equivalent to one of the shape

$$
\widehat{P}_{n / 2}\left(\left(x-\frac{1}{2}\right)^{2}\right)
$$

where $\widehat{P}_{n / 2}(x)$ is a polynomial of degree $n / 2$ which is indecomposable for every $n$. These results from [6] and [11] suggest the following notion. We say that an Appell sequence $\left\{P_{n}(x)\right\}_{n \geq 0}$ is of special type if $P_{n}(x)$ is indecomposable over $\mathbb{C}$ for all odd $n$, and, for even $n$, every nontrivial decomposition of $P_{n}(x)$ is equivalent to a decomposition of the form

$$
P_{n}(x)=\widehat{P}_{n / 2}\left(\left(x-\frac{1}{2}\right)^{2}\right)
$$


with an indecomposable polynomial $\widehat{P}_{n / 2}(x)$ over $\mathbb{C}$ of degree $n / 2$. Clearly, the polynomials $\left\{B_{n}(x)\right\}_{n>0}$ and $\left\{E_{n}(x)\right\}_{n>0}$ are of special type.

The theory of polynomial decomposition is strongly connected to the theory of separable Diophantine equations since, in 2000, Bilu and Tichy [5] established their general ineffective finiteness criterion on equations of the form $f(x)=g(y)$. (See Proposition 1 below.)

In this paper we study the Diophantine equation

$$
P_{n}(x)=g(y) \text { in integers } x, y,
$$

where $P_{n}(x)$ is from an Appell sequence of special type and $g(x) \in \mathbb{Q}[x]$, $\operatorname{deg} g(x) \geq 3$. For technical reasons, we restrict ourselves to Appell sequences $\left\{P_{n}(x)\right\}_{n \geq 0}$ for which

$$
\frac{3 P_{2}\left(-c_{1} / c_{0}\right)^{2}-2 c_{0} P_{4}\left(-c_{1} / c_{0}\right)}{3 P_{2}\left(-c_{1} / c_{0}\right)^{2}-c_{0} P_{4}\left(-c_{1} / c_{0}\right)} \quad \text { is not a positive integer. }
$$

Remark. In the following table, we give the value of the constant from (6) for the case when $P_{n}(x)$ is a Bernoulli, Euler or an Hermite polynomial, respectively.

$$
\begin{array}{c|c|c}
B_{n}(x) & E_{n}(x) & H_{n}(x) \\
\hline 9 / 2 & 7 / 2 & \text { undefined }
\end{array}
$$

For $P_{n}(x)=B_{n}(x)$, Rakaczki [10], and independently Kulkarni and Sury [9] characterized those pairs $(n, g(y))$ for which equation (5) has infinitely many integer solutions. Recently, Rakaczki and Kreso [11] proved an analogous result for the case when $P_{n}(x)=\left(E_{n}(0) \pm E_{n}(x)\right) / 2$ (which is not an Appell polynomial anymore). For further related results we refer to $[7,8]$.

We prove the following.

Theorem 1. Let $g(x) \in \mathbb{Q}[x]$ with $\operatorname{deg} g(x) \geq 3$, and suppose that $\left\{P_{n}(x)\right\}_{n \geq 0}$ is an Appell sequence of special type with property (6). Then for $n \geq 7$, equation (5) has only finitely many integer solutions $x, y$, apart from the following cases:

(i) $g(x)=P_{n}(h(x))$, where $h(x)$ is a polynomial over $\mathbb{Q}$.

(ii) $g(x)=\gamma\left(\delta(x)^{m}\right)$, where $m$ is a positive integer.

(iii) $n$ is even and $g(x)=\widehat{P}_{n / 2}\left(q(x)^{2}\right)$

(iv) $n$ is even and $g(x)=\widehat{P}_{n / 2}\left(\delta(x) q(x)^{2}\right)$

(v) $n$ is even and $g(x)=\widehat{P}_{n / 2}\left(c \delta(x)^{t}\right)$, where $t \geq 3$ is an odd integer

(vi) $n$ is even and $g(x)=\widehat{P}_{n / 2}\left(\left(a \delta(x)^{2}+b\right) q(x)^{2}\right)$

Here $a, b, c \in \mathbb{Q} \backslash\{0\}, \gamma(x), \delta(x) \in \mathbb{Q}[x]$ are linear polynomials and $q(x) \in \mathbb{Q}[x]$ is a non-zero polynomial. 
We prove the above theorem by applying among other things the general finiteness criterion of Bilu and Tichy [5] for equation (5). Hence our finiteness result is ineffective.

Remark. For $n \geq 7$, our main result is a common generalization of the mentioned results of Rakaczki [10], Kulkarni and Sury [9] and Rakaczki and Kreso [11]. In the special cases $P_{n}(x) \in\left\{B_{n}(x), E_{n}(x)\right\}$, one can exclude the exceptional case (ii) by making use of some specific properties of the Bernoulli or Euler polynomials, respectively. (See $[9,10,11])$

\section{Auxiliary Results}

Before proving Theorem 1, we collect those results that we are going to apply in the proof. First, we recall the finiteness criterion of Bilu and Tichy [5]. To do this, we need to define five kinds of so-called standard pairs of polynomials.

Let $\alpha, \beta$ be nonzero rational numbers, $\mu, \nu, q>0$ and $r \geq 0$ be integers, and let $v(x) \in \mathbb{Q}[x]$ be a nonzero polynomial (which may be constant). Denote by $D_{\mu}(x, \delta)$ the $\mu$-th Dickson polynomial, defined by the functional equation $D_{\mu}(z+\delta / z, \delta)=z^{\mu}+(\delta / z)^{\mu}$ or by the explicit formula

$$
D_{\mu}(x, \delta)=\sum_{i=0}^{\lfloor\mu / 2\rfloor} d_{\mu, i} x^{\mu-2 i} \quad \text { with } \quad d_{\mu, i}=\frac{\mu}{\mu-i}\left(\begin{array}{c}
\mu-i \\
i
\end{array}\right)(-\delta)^{i} .
$$

Two polynomials $f_{1}(x)$ and $g_{1}(x)$ are said to form a standard pair over $\mathbb{Q}$ if one of the ordered pairs $\left(f_{1}(x), g_{1}(x)\right)$ or $\left(g_{1}(x), f_{1}(x)\right)$ belongs to the list below. The five kinds of standard pairs are then listed in the following table.

\begin{tabular}{|l|l|l|}
\hline kind & explicit form of $\left\{f_{1}(x), g_{1}(x)\right\}$ & parameter restrictions \\
\hline first & $\left(x^{q}, \alpha x^{r} v(x)^{q}\right)$ & $\begin{array}{l}0 \leq r<q,(r, q)=1, \\
r+\operatorname{deg} v(x)>0\end{array}$ \\
\hline second & $\left(x^{2},\left(\alpha x^{2}+\beta\right) v(x)^{2}\right)$ & - \\
\hline third & $\left(D_{\mu}\left(x, \alpha^{\nu}\right), D_{\nu}\left(x, \alpha^{\mu}\right)\right)$ & $(\mu, \nu)=1$ \\
\hline fourth & $\left(\alpha^{\frac{-\mu}{2}} D_{\mu}(x, \alpha),-\beta^{\frac{-\nu}{2}} D_{\nu}(x, \beta)\right)$ & $(\mu, \nu)=2$ \\
\hline fifth & $\left(\left(\alpha x^{2}-1\right)^{3}, 3 x^{4}-4 x^{3}\right)$ & - \\
\hline
\end{tabular}

The following proposition is a special case of the main result of [5].

Proposition 1. Let $f(x), g(x) \in \mathbb{Q}[x]$ be nonconstant polynomials such that the equation $f(x)=g(y)$ has infinitely many solutions in rational integers $x, y$. Then $f=\varphi \circ f_{1} \circ \lambda$ and $g=\varphi \circ g_{1} \circ \mu$, 
where $\lambda(x), \mu(x) \in \mathbb{Q}[x]$ are linear polynomials, $\varphi(x) \in \mathbb{Q}[x]$, and $\left(f_{1}(x), g_{1}(x)\right)$ is a standard pair over $\mathbb{Q}$.

For $P(x) \in \mathbb{C}[x]$, a complex number $c$ is said to be an extremum if $P(x)-c$ has multiple roots. The $P$-type of $c$ is defined to be the tuple $\left(\alpha_{1}, \ldots, \alpha_{s}\right)$ of the multiplicities of the distinct roots of $P(x)-c$ in an increasing order. Obviously, $s<\operatorname{deg} P(x)$ and $\alpha_{1}+\ldots+\alpha_{s}=\operatorname{deg} P(x)$.

Proposition 2. For $a \neq 0$ and $k \geq 3, D_{\mu}(x, \alpha)$ has exactly two extrema $\pm 2 \alpha^{\frac{\mu}{2}}$. If $\mu$ is odd, then both are of P-type $(1,2,2, \ldots, 2)$. If $\mu$ is even, then $2 \alpha^{\frac{\mu}{2}}$ is of P-type $(1,1,2, \ldots, 2)$ and $-2 \alpha^{\frac{\mu}{2}}$ is of P-type $(2,2, \ldots, 2)$.

Proof. See, for instance [4, Proposition 3.3].

We end this section with two technical results. Let $d_{1}, e_{1} \in \mathbb{Q}^{*}$ and $d_{0}, e_{0} \in \mathbb{Q}$

Proposition 3. Suppose that $\left\{P_{n}(x)\right\}_{n \geq 0}$ is an Appell sequence of special type. Then the polynomial $P_{n}\left(d_{1} x+d_{0}\right)$ is not of the form $e_{1} x^{q}+e_{0}$, with $q \geq 7$.

Proof. We assume the contrary, i.e., that we have

$$
P_{n}\left(d_{1} x+d_{0}\right)=e_{1} x^{q}+e_{0}
$$

with $q \geq 7$. Obviously, we then have $n=q$.

We observe from (2) and (7) that

$$
P_{1}\left(d_{0}\right)=P_{2}\left(d_{0}\right)=\ldots=P_{n-1}\left(d_{0}\right)=0 .
$$

Since, by (3), $P_{1}\left(d_{0}\right)=c_{0} d_{0}+c_{1}$, we get

$$
d_{0}=-\frac{c_{1}}{c_{0}} \text {. }
$$

Further, since, by (1),

$$
P_{k}(x)=\frac{k !}{(n-1) !} P_{n-1}^{(n-1-k)}(x), \quad k=1, \ldots, n-1,
$$

we infer that $d_{0}$ is a root of $P_{n-1}(x)$ of multiplicity $(n-1)$. Thus, in view of (9), we have $P_{n-1}(x)=c_{0}\left(x+c_{1} / c_{0}\right)^{n-1}$, which implies

$$
P_{n}(x)=c_{0}\left(x+\frac{c_{1}}{c_{0}}\right)^{n}+C \quad \text { with } \quad C=P_{n}\left(-\frac{c_{1}}{c_{0}}\right) .
$$

First, if $n \geq 7$ is even, then, by (11), one can easily find the nontrivial decomposition $P_{n}(x)=Q(R(x))$ with

$$
Q(x)=c_{0} x^{2}+C, \quad \text { and } \quad R(x)=\left(x+\frac{c_{1}}{c_{0}}\right)^{n / 2} .
$$


Since $n \geq 7$, this nontrivial decomposition is obviously not equivalent to the one in (4), contradicting that $\left\{P_{n}(x)\right\}_{n \geq 0}$ is of special type.

Now, let $n \geq 7$ be an odd positive integer. If $n$ is composite, then any divisor $v$ of $n$ with $1<v<n$ leads to a nontrivial decomposition

$$
P_{n}(x)=c_{0}\left(\left(x+\frac{c_{1}}{c_{0}}\right)^{v}\right)^{n / v}+C,
$$

which again contradicts that $\left\{P_{n}(x)\right\}_{n \geq 0}$ is of special type (and in this case $P_{n}(x)$ is indecomposable). If $n$ is a prime, then derivating both sides of (11) we obtain

$$
P_{n-1}(x)=c_{0}\left(x-\frac{1}{2}\right)^{n-1},
$$

where of course the exponent $n-1$ is even. Similarly as above, this leads to a nontrivial decomposition not equivalent to (4) and thus to a contradiction.

Proposition 4. Suppose that $\left\{P_{n}(x)\right\}_{n>0}$ is an Appell sequence which satisfies (6). Then the polynomial $P_{n}\left(d_{1} x+d_{0}\right)$ is not of the form $e_{1} D_{\mu}(x, \delta)+e_{0}$, where $D_{\mu}(x, \delta)$ the $\mu$-th Dickson polynomial with $\mu>4$, $\delta \in \mathbb{Q}^{*}$.

Proof. Suppose that the Appell sequence $\left\{P_{n}(x)\right\}_{n \geq 0}$ satisfies (6), and that we have

$$
P_{n}\left(d_{1} x+d_{0}\right)=e_{1} D_{\mu}(x, \delta)+e_{0} .
$$

Clearly, $n=\mu$. Comparing the leading coefficients of both sides we get

$$
d_{1}^{n} c_{0}=e_{1},
$$

where the numbers $c_{k}(k \geq 0)$ are defined in (3). Similarly, from (2) and the equality of the coefficients of $x^{n-1}$ on both sides we obtain

$$
n d_{1}^{n-1} P_{1}\left(d_{0}\right)=0
$$

which implies

$$
d_{0}=-\frac{c_{1}}{c_{0}}
$$

Again, by (2), comparing the coefficients of $x^{n-2}$ gives

$$
\left(\begin{array}{l}
n \\
2
\end{array}\right) d_{1}^{n-2} P_{2}\left(d_{0}\right)=-e_{1} n \delta
$$

whence, together with (16) it follows that

$$
d_{1}^{2}=-\frac{(n-1) P_{2}\left(d_{0}\right)}{2 c_{0} \delta}
$$


Now we compare the coefficients of $x^{n-4}$ on both sides of (15) and we obtain

$$
\left(\begin{array}{l}
n \\
4
\end{array}\right) d_{1}^{n-4} P_{4}\left(d_{0}\right)=\frac{e_{1} n(n-3) \delta^{2}}{2},
$$

which along with (16) leads to

$$
d_{1}^{4}=\frac{(n-1)(n-2) P_{4}\left(d_{0}\right)}{12 c_{0} \delta^{2}} .
$$

After substituting (20) into (22), we obtain

$$
3(n-1) P_{2}\left(d_{0}\right)^{2}=(n-2) c_{0} P_{4}\left(d_{0}\right),
$$

whence, together with (18) it follows that

$$
n=\frac{3 P_{2}\left(-c_{1} / c_{0}\right)^{2}-2 c_{0} P_{4}\left(-c_{1} / c_{0}\right)}{3 P_{2}\left(-c_{1} / c_{0}\right)^{2}-c_{0} P_{4}\left(-c_{1} / c_{0}\right)} .
$$

This is a contradiction by (6).

We note that Proposition 4 is a common generalization of Lemma 5.3 in [6], Lemma 2.4 in [3], and of the second statement of Lemma 12 in $[11]$.

\section{Proof of Theorem 1}

Let $g(x) \in \mathbb{Q}[x]$ with $\operatorname{deg} g(x) \geq 3$. Suppose that equation (5) has infinitely many integer solutions $x, y$ with an Appell sequence $\left\{P_{n}(x)\right\}_{n \geq 0}$ of special type satisfying (6) and with $n \geq 7$. Then by Proposition 1 it follows that there exist $\lambda(x), \mu(x), \varphi(x) \in \mathbb{Q}[x]$, $\operatorname{deg} \lambda(x)=\operatorname{deg} \mu(x)=1$ such that

$$
P_{n}(x)=\varphi\left(f_{1}(\lambda(x))\right) \quad \text { and } \quad g(x)=\varphi\left(g_{1}(\mu(x))\right),
$$

where $\left(f_{1}(x), g_{1}(x)\right)$ is a standard pair over $\mathbb{Q}$.

Let $\lambda^{-1}(x)=a_{1} x+a_{0}, \mu^{-1}(x)=b_{1} x+b_{0}$, where $a_{0}, a_{1}, b_{0}, b_{1} \in \mathbb{Q}$ with $a_{1} b_{1} \neq 0$. Then we can rewrite $(25)$ as

$$
P_{n}\left(a_{1} x+a_{0}\right)=\varphi\left(f_{1}(x)\right) \quad \text { and } \quad g\left(b_{1} x+b_{0}\right)=\varphi\left(g_{1}(x)\right),
$$

Since $P_{n}(x)$ is of special type and $\operatorname{deg} P_{n}(x)=n$, we obtain that

$$
\operatorname{deg} \varphi(x) \in\left\{1, \frac{n}{2}, n\right\} .
$$


3.1. The case $\operatorname{deg} \varphi(x)=n$. If we assume that $\operatorname{deg} \varphi(x)=n$, then by (25), we have $\operatorname{deg} f_{1}(x)=1$. Thus $P_{n}(x)=\varphi(t(x))$, where $t(x) \in \mathbb{Q}[x]$ is a linear polynomial. Clearly, $t^{-1}(x) \in \mathbb{Q}[x]$ is also linear. By $(25)$, we obtain $P_{n}\left(t^{-1}(x)\right)=\varphi\left(t\left(t^{-1}(x)\right)\right)=\varphi(x)$. Hence

$$
g(x)=\varphi\left(g_{1}(\mu(x))\right)=P_{n}\left(t^{-1}\left(g_{1}(\mu(x))\right)\right)=P_{n}(q(x)),
$$

where $q(x)=t^{-1}\left(g_{1}(\mu(x))\right)$. So, if, in our case, equation (5) has infinitely many solutions, then $g(x)$ is of the form as in Theorem 1 (i).

3.2. The case $\operatorname{deg} \varphi(x)=1$. Let $\varphi(x)=\varphi_{1} x+\varphi_{0}$, where $\varphi_{1}, \varphi_{0} \in \mathbb{Q}$ and $\varphi_{1} \neq 0$. We study now the five kinds of standard pairs.

In view of our assumptions on $n$ and $\operatorname{deg} g(x)$, it follows that the standard pair $\left(f_{1}(x), g_{1}(x)\right)$ cannot be of the second or fifth kind.

If it is of the third or fourth kind, we then have $P_{n}\left(a_{1} x+a_{0}\right)=$ $e_{1} D_{\mu}(x, \delta)+e_{0}$ with $e_{0} \in \mathbb{Q}, e_{1}, \delta \in \mathbb{Q}^{*}$. This contradicts Proposition 4 .

If $\left(f_{1}(x), g_{1}(x)\right)$ is a standard pair of the first kind, then we have either

(I) $P_{n}\left(a_{1} x+a_{0}\right)=\varphi_{1} x^{q}+\varphi_{0}$, or

(II) $P_{n}\left(a_{1} x+a_{0}\right)=\varphi_{1} \alpha x^{p} \nu(x)^{q}+\varphi_{0}$, where $0 \leq p<q,(p, q)=1$ and $p+\operatorname{deg} \nu(x)>0$.

The first case (I) is impossible by Proposition 3 since $n \geq 7$ by assumption.

Let us now consider the second case (II). Then we have $g(x)=$ $\varphi_{1} \mu(x)^{q}+\varphi_{0}=\varphi\left(\mu(x)^{q}\right)$, where $q \geq 3$ and $\mu(x) \in \mathbb{Q}[x]$ is linear, which is case (ii) of Theorem 1.

3.3. The case $\operatorname{deg} \varphi(x)=n / 2$. Clearly, $n$ is then even, and from (25) we observe that $\operatorname{deg} f_{1}(x)=2$. Hence it follows that, in (25), $\left(f_{1}(x), g_{1}(x)\right)$ cannot be a standart pair of the fifth kind. Further, we obtain a nontrivial decomposition of $P_{n}(x)$, which, since $P_{n}(x)$ is of special type, implies that there exists a linear polynomial $\ell(x)=\ell_{1} x+\ell_{0}$ over $\mathbb{Q}$ such that

$$
\varphi(x)=\widehat{P}_{n / 2}(\ell(x)) \quad \text { and } \quad \ell\left(f_{1}(\lambda(x))\right)=\left(x-\frac{1}{2}\right)^{2} .
$$

Again, we study the remaining kinds of standard pairs.

First, we consider the case when, in $(25),\left(f_{1}(x), g_{1}(x)\right)$ is a standard pair of the first kind. If $f_{1}(x)=x^{t}$, then by $\operatorname{deg} f_{1}(x)=2$, we have $\left(f_{1}(x), g_{1}(x)\right)=\left(x^{2}, \alpha x p(x)^{2}\right)$. Putting $\lambda(x)=\lambda_{1} x+\lambda_{0}$, (28) takes the form $\ell\left(\left(\lambda_{1} x+\lambda_{0}\right)^{2}\right)=(x-1 / 2)^{2}$, whence an easy calculation gives 
$\ell(x)=x / \lambda_{1}^{2}$. Substituting this to (25), we obtain

$$
g(x)=\widehat{P}_{n / 2}\left(\ell\left(g_{1}(\mu(x))\right)\right)=\widehat{P}_{n / 2}\left(\frac{\alpha \mu(x) p(\mu(x))^{2}}{\lambda_{1}^{2}}\right)
$$

So $g(x)$ is of the form (iv) with $\delta(x)=\alpha \mu(x) / \lambda_{1}^{2}$ and $q(x)=p(\mu(x))$.

In the switched case $\left(f_{1}(x), g_{1}(x)\right)=\left(\alpha x^{r} p(x)^{t}, x^{t}\right)$, where $0 \leq r<t$, $(r, t)=1$ and $r+\operatorname{deg} p(x)>0, \operatorname{deg} f_{1}(x)=2$ implies that one of the following cases occurs:

(A) $r=0, t=1$ and $\operatorname{deg} p(x)=2$, or

(B) $r=2, t>2$ is odd and $p(x)$ is a constant polynomial.

In case (A) we have $g_{1}(x)=x$, whence from (25) and (28) we obtain

$$
g(x)=\widehat{P}_{n / 2}\left(\ell\left(g_{1}(\mu(x))\right)\right)=\widehat{P}_{n / 2}(\ell(\mu(x)))=\widehat{P}_{n / 2}\left(\delta(x) q(x)^{2}\right),
$$

where $\delta(x)=\ell(\mu(x))$ and $q(x) \equiv 1$. Thus $g(x)$ is again of the form (iv).

In the second case $(\mathrm{B})$, we can write $f_{1}(x)=\beta x^{2}$, with $\beta=\alpha p(x)^{t} \in$ $\mathbb{Q} \backslash\{0\}$. Substituting this to $(28)$, we deduce that $\ell(x)=x /\left(\beta \lambda_{1}^{2}\right)$, whence, by (25), we get

$$
g(x)=\widehat{P}_{n / 2}\left(\ell\left(g_{1}(\mu(x))\right)\right)=\widehat{P}_{n / 2}\left(\frac{\mu(x)^{t}}{\beta \lambda_{1}^{2}}\right)=\widehat{P}_{n / 2}\left(c \delta(x)^{t}\right),
$$

where $c=1 /\left(\beta \lambda_{1}^{2}\right), \delta(x)=\mu(x)$ and $t>2$ is odd. This is option (v) in Theorem 1 .

Next let, in (25), be a the standard pair $\left(f_{1}(x), g_{1}(x)\right)$ of the second kind. If $\left(f_{1}(x), g_{1}(x)\right)=\left(x^{2},\left(\alpha x^{2}+\beta\right) v(x)^{2}\right)$, then a calculation from (28) yields $\ell(x)=x / \lambda_{1}^{2}$, and by (25) we have

$$
\begin{aligned}
& g(x)=\widehat{P}_{n / 2}\left(\ell\left(g_{1}(\mu(x))\right)\right)= \\
& =\widehat{P}_{n / 2}\left(\frac{\left(\alpha \mu(x)^{2}+\beta\right) v(\mu(x))^{2}}{\lambda_{1}^{2}}\right)=\widehat{P}_{n / 2}\left(\left(\alpha \delta(x)^{2}+\beta\right) q(x)^{2}\right),
\end{aligned}
$$

where $\delta(x)=\mu(x)$ and $q(x)=v(\mu(x)) / \lambda_{1}$. So we are led to option (vi) of our theorem.

In the switched case $\left(f_{1}(x), g_{1}(x)\right)=\left(\left(\alpha x^{2}+\beta\right) v(x)^{2}, x^{2}\right)$, since $\operatorname{deg} f_{1}(x)=2, v(x)$ is a constant polynomial and

$$
g(x)=\widehat{P}_{n / 2}\left(\ell\left(g_{1}(\mu(x))\right)\right)=\widehat{P}_{n / 2}\left(\left(\ell_{1} \mu(x)^{2}+\ell_{0}\right) q(x)^{2}\right),
$$

where $q(x) \equiv 1$. Thus, we arrived again at option (vi) with $\delta(x)=\mu(x)$ and $a=\ell_{1}, b=\ell_{0}$.

Now, if the standard pair $\left(f_{1}(x), g_{1}(x)\right)$ is of the third kind over $\mathbb{Q}$, then $\left(f_{1}(x), g_{1}(x)\right)=\left(D_{2}\left(x, \alpha^{t}\right), D_{t}\left(x, \alpha^{2}\right)\right)$ with $t$ being odd. Let us 
substitute $f_{1}(x)=x^{2}-2 \alpha^{t}$ into $(28)$ to deduce that $\ell(x)=\left(x+2 \alpha^{t}\right) / \lambda_{1}^{2}$, whence

$$
g(x)=\widehat{P}_{n / 2}\left(\ell\left(g_{1}(\mu(x))\right)\right)=\widehat{P}_{n / 2}\left(\frac{D_{t}\left(\mu(x), \alpha^{2}\right)+2 \alpha^{t}}{\lambda_{1}^{2}}\right) .
$$

It follows from Proposition 2 that $-2 \alpha^{t} / \lambda_{1}^{2}$ is an extremum of the polynomial $D_{t}\left(\mu(x), \alpha^{2}\right) / \lambda_{1}^{2}$, which is of $P$-type $(1,2, \ldots, 2)$ as $t$ is odd. Hence $\left(D_{t}\left(\mu(x), \alpha^{2}\right)+2 \alpha^{t}\right) / \lambda_{1}^{2}=\delta(x) q(x)^{2}$ for some $\delta(x), q(x) \in \mathbb{Q}[x]$ with $\operatorname{deg} \delta(x)=1$. We deduce, that $g(x)$ is of the form (iv).

Finally, consider the case when $\left(f_{1}(x), g_{1}(x)\right)$ is a standard pair of the fourth kind over $\mathbb{Q}$. Then

$$
\left(f_{1}(x), g_{1}(x)\right)=\left(\frac{D_{2}(x, \alpha)}{\alpha}, \frac{D_{t}(x, \beta)}{\beta^{(t / 2)}}\right)
$$

with an even $t$. Substituting this into (28), an easy calculation yields $\ell(x)=(\alpha x+2 \alpha) / \lambda_{1}^{2}$, whence, by (25), we obtain

$$
g(x)=\widehat{P}_{n / 2}\left(\ell\left(g_{1}(\mu(x))\right)\right)=\widehat{P}_{n / 2}\left(\frac{\alpha \beta^{-t / 2} D_{t}(\mu(x), \beta)+2 \alpha}{\lambda_{1}^{2}}\right) .
$$

Now from Proposition 2 we infer that

$$
-\frac{2 \beta^{t / 2} \alpha \beta^{-t / 2}}{\lambda_{1}^{2}}=-\frac{2 \alpha}{\lambda_{1}^{2}}
$$

is one of the two extrema of the polynomial $\alpha \beta^{-t / 2} D_{t}(\mu(x), \beta) /\left(\lambda_{1}^{2}\right)$ and it is of $P$-type $(2,2, \ldots, 2)$ as $t$ is even. Therefore we have

$$
\frac{\alpha \beta^{-t / 2} D_{t}(\mu(x), \beta)+2 \alpha}{\lambda_{1}^{2}}=q(x)^{2}
$$

for some $q(x) \in \mathbb{Q}[x]$. Thus $g(x)$ is of the form (iii), which completes the proof.

\section{ACKNowledGements}

The first author was supported by the Hungarian Academy of Sciences and by the OTKA grant NK104208. The second author was partially supported by the European Union and the European Social Fund through project Supercomputer, the national virtual lab (grant no: TÁMOP-4.2.2.C-11/1/KONV-2012-0010). 


\section{REFERENCES}

1. M. Abramowitz and I.A. Stegun, Handbook of Mathematical Functions, National Bureau of Standards, 1964.

2. P. Appell, Sur une classe de polynômes. Ann. Sci. Ecole Norm. Sup., 9 (1880), 119-144.

3. A. Bazsó, D. Kreso, F. LuCa and Á. Pintér, On equal values of power sums of arithmetic progressions, Glas. Mat. Ser. III , 47 (2012), 253-263.

4. Y. F. Bilu, Quadratic factors of $f(x)-g(y)$, Acta Arith., 90 (1999), 341-355.

5. Y. F. Bilu and R. F. Tichy, The Diophantine equation $f(x)=g(y)$, Acta Arith., 95 (2000), 261-288.

6. Y. F. Bilu, B. Brindza, P. Kirschenhofer, Á. Pintér and R. F. Tichy, Diophantine equations and Bernoulli polynomials (with an Appendix by A. Schinzel), Compositio Math., 131 (2002), 173-188.

7. M. KulKarni and B. Sury, Diophantine equations with Bernoulli polynomials, Acta Arith., 116 (2005), 25-34.

8. M. KulKarni and B. SurY, A class of Diophantine equations involving Bernoulli polynomials, Indag. Math. (N.S.), 16 (2005), 51-65.

9. M. KulKarni and B. SurY, On the Diophantine equation $1+x+\frac{x^{2}}{2 !}+\ldots+$ $\frac{x^{n}}{n !}=g(y)$. . In: Diophantine equations, 121-134, Tata Inst. Fund. Res. Stud. Math., Mumbai, 2008.

10. Cs. Rakaczki, On the Diophantine equation $S_{m}(x)=g(y)$, Publ. Math. Debrecen, 65 (2004), 439-460.

11. Cs. RakaczKi and D. Kreso, Diophantine equations with Euler polynomials, Acta Arith., 161 (2013), 267-281.

12. S. Roman, The umbral calculus, Academic Press, New York, 1984.

ANDRÁs BAzsó

InSTITUTE OF MATHEMATICS

MTA-DE Research Group "Equations Functions and Curves" Hungarian Academy of Sciences and University of Debrecen

P.O. Box 12, H-4010 Debrecen, Hungary

E-mail address: bazsoa@science.unideb.hu

IsTVÁN PINK

Institute of Mathematics

UNIVERSITY OF DEBRECEN

P.O. Box 12, H-4010 Debrecen, Hungary

E-mail address: pinki@science.unideb.hu 